\title{
Metabolic and Molecular Imaging of Atherosclerosis and Venous Thromboembolism
}

\author{
Eric A. Osborn*1,2, Chase W. Kessinger*1 ${ }^{1}$, Ahmed Tawakol ${ }^{1}$, and Farouc A. Jaffer ${ }^{1}$ \\ ${ }^{I}$ Cardiovascular Research Center, Cardiology Division, Massachusetts General Hospital, Harvard Medical School, Boston, \\ Massachusetts; and ${ }^{2}$ Cardiology Division, Beth Israel Deaconess Medical Center, Harvard Medical School, Boston, Massachusetts
}

Prediction is very difficult, especially if it's about the future.

-Niels Bohr

Metabolic and molecular imaging continues to advance our
understanding of vascular disease pathophysiology. At present,
${ }^{18}$ F-FDG PET imaging is the most widely used clinical tool for
metabolic and molecular imaging of atherosclerosis. However,
novel nuclear tracers and intravascular optical near-infrared fluo-
rescence imaging catheters are emerging to assess new biologic
targets in vivo and in coronary arteries. This review highlights
current metabolic and molecular imaging clinical and near-clinical
applications within atherosclerosis and venous thromboembo-
lism, and explores the potential for metabolic and molecular im-
aging to affect patient-level risk prediction and disease treatment.

Key Words: molecular imaging; atherosclerosis; near infrared fluorescence; PET; venous thromboembolism

J Nucl Med 2017; 58:871-877

DOI: 10.2967/jnumed.116.182873

I this focused review, we showcase state-of-the-art molecular imaging applications to atherosclerosis and venous thromboembolism (VTE). Clinical or near-clinical applications will be highlighted, illuminating how molecular imaging can provide insights into disease pathophysiology, risk prediction, and management.

\section{ATHEROSCLEROSIS}

\section{Background}

Atherosclerosis is the primary cause of heart attack, stroke, and limb ischemia and remains a leading cause of death worldwide. Clinical atherosclerosis imaging traditionally assesses anatomic measures such as luminal stenosis. However, imaging of structural features provides limited insights into the pathobiology central to atherosclerosis

Received Dec. 16, 2016; revision accepted Apr. 19, 2017.

For correspondence or reprints contact: Farouc A. Jaffer, Cardiovascular Research Center, Cardiology Division, Massachusetts General Hospital,

Simches Research Bldg. \#3206, Boston, MA 02114.

E-mail: fjaffer@mgh.harvard.edu

${ }^{*}$ Contributed equally to this work.

Published online Apr. 27, 2017.

COPYRIGHT (c) 2017 by the Society of Nuclear Medicine and Molecular Imaging. disease progression, which is a biologic consequence of accumulated inflammatory cells and oxidized lipids within the vessel wall. Molecular imaging is a complementary approach that provides new insights into mechanisms of atherosclerosis progression and complications. For larger arteries (carotid, iliac, aorta), ${ }^{18}$ F-FDG PET imaging currently predominates to detect plaque inflammatory cells (macrophages). In the smaller-sized coronary arteries, ${ }^{18} \mathrm{~F}$-sodium fluoride $\left({ }^{18} \mathrm{~F}-\mathrm{NaF}\right)$ PET and highresolution intravascular near-infrared fluorescence (NIRF) molecular imaging are emerging new approaches to image plaque mineralization and inflammation (e.g., protease activity), respectively.

\section{F-FDG PET}

In atherosclerosis, the most widely used PET tracer is ${ }^{18} \mathrm{~F}-\mathrm{FDG}$, a glucose analog consumed by metabolically active cells. ${ }^{18} \mathrm{~F}$-FDG possesses a 110 -min half-life and maximum 2.4-mm positron range. After phosphorylation, trapped intracellular ${ }^{18}$ F-FDG-6-phosphate cannot proceed down glycolytic pathways and accumulates in direct relation to the degree of cellular glycolysis, substantially reflecting plaque macrophage content.

Association with Cardiovascular Risk Factors and Prediction of Cardiovascular Events. Atherosclerosis ${ }^{18} \mathrm{~F}-\mathrm{FDG}$ PET metabolic imaging is positioned to improve risk prediction beyond structural imaging measures, by predicting atheroma progression and subsequent clinical events, and to tailor atheroma pharmacotherapies.

Risk factors (e.g., smoking, diabetes mellitus, hyperlipidemia, hypertension) are established predictors of cardiovascular events on a population-based level. To assess relationships between cardiac risk factors and ${ }^{18} \mathrm{~F}-\mathrm{FDG}$ PET, Noh et al. measured ${ }^{18} \mathrm{~F}-\mathrm{FDG}$ carotid plaque uptake in 1,181 asymptomatic subjects, and correlated the results with 10-y Framingham risk score (FRS) (mean \pm SD, $12.3 \% \pm 8.7 \%$, indicating intermediate risk) $(1)$. The authors determined that an elevated carotid plaque ${ }^{18} \mathrm{~F}-\mathrm{FDG}$ uptake (mean target-to-background ratio $\geq 1.7$ ) increased the chance of having an elevated FRS of $10 \%$ or more (odds ratio, 1.9). ${ }^{18} \mathrm{~F}-\mathrm{FDG}$ uptake has also been associated with other risk factors. One study explored the relationship 
between plaque inflammation (by ${ }^{18} \mathrm{~F}$ PET) and atherogenic lipoprotein(a) (Lp(a)), a proinflammatory oxidized phospholipid transporter associated with accelerated arterial inflammation. Compared with 30 matched subjects with normal $\mathrm{Lp}$ (a) levels, those with elevated $\mathrm{Lp}$ (a) manifested greater arterial inflammation (2).

Beyond risk factors, imaging arterial inflammation may provide direct insights into atherosclerosis progression. Cho et al. recently observed that aortic ${ }^{18}$ F-FDG PET uptake predicted the progression of coronary calcification at $1 \mathrm{y}$ (3). In 96 subjects without known coronary disease or statin use, elevated aortic ${ }^{18}$ F-FDG uptake significantly associated with incident coronary calcification (odds ratio, 4.39; $P=$ 0.007). Similarly, Abdelbaky et al. demonstrated that local ${ }^{18} \mathrm{~F}$-FDG uptake predicts incident calcium deposition in the underlying atheroma (4).

More importantly, several retrospective studies have shown that aortic ${ }^{18}$ F-FDG uptake independently predicts cardiovascular disease (CVD) risk above the FRS (5), and evolving data support prospective ${ }^{18}$ F-FDG PET imaging. ${ }^{18}$ F-FDG PET carotid arterial inflammation independently predicted recurrent ipsilateral cerebrovascular events in 60 patients with recent stroke (6). Accordingly, ${ }^{18}$ F-FDG PET/ CT arterial imaging may provide additive prognostic information beyond risk factor assessment and structural imaging data.

${ }^{18}$ F-FDG PET imaging has yielded insights into how adipose tissue, particularly visceral adiposity, associates with heightened cardiovascular risk. Figueroa et al. evaluated CT adipose tissue, ${ }^{18} \mathrm{~F}$-FDG PET arterial inflammation, and cardiovascular events in 415 asymptomatic patients (mean body mass index, $26.4 \mathrm{~kg} / \mathrm{m}^{2}$ ) (7). Visceral fat volume correlated positively with ${ }^{18} \mathrm{~F}$-FDG inflammation $(r=$ $0.290, P<0.001)$. Over $4 \mathrm{y}$, the visceral fat volume associated with increased cardiovascular event risk (hazard ratio, 1.15, and $P<0.001$; hazard ratio, 3.60, and $P<0.001$, respectively). In a separate 443 -patient study, ${ }^{18}$ F-FDG PET signal in plaques and brown adipose tissue, a metabolically active tissue present in childhood that regresses with age, demonstrated that brown fat ${ }^{18} \mathrm{~F}-\mathrm{FDG}$ activity correlated negatively with ${ }^{18} \mathrm{~F}$-FDG arterial inflammation $(r=-0.147$, $P<0.01)(8)$. Brown fat ${ }^{18} \mathrm{~F}-\mathrm{FDG}$ uptake was further associated with improved cardiac event-free survival $(P=0.048)$. These data support the notion that visceral fat spurs arterial inflammation and subsequent events and that brown fat may be protective.

Patients with acute coronary syndrome (ACS) harbor a heightened risk of repeated cardiovascular events. Pathophysiologically, recent preclinical mechanistic studies support splenic and bone marrow (the cardiospenic axis) activation as mediators of activated leukocyte trafficking and subsequent atheroma progression. Emami et al. imaged 22 ACS patients, demonstrating increased ${ }^{18} \mathrm{~F}-\mathrm{FDG}$ spleen uptake compared with non-ACS controls (Fig. 1; SUV, $2.6 \pm$ 0.6 vs. $2.1 \pm 0.3 ; P=0.03$ ) (9). A 464-patient substudy revealed that subjects with increased ${ }^{18} \mathrm{~F}-\mathrm{FDG}$ splenic ac-

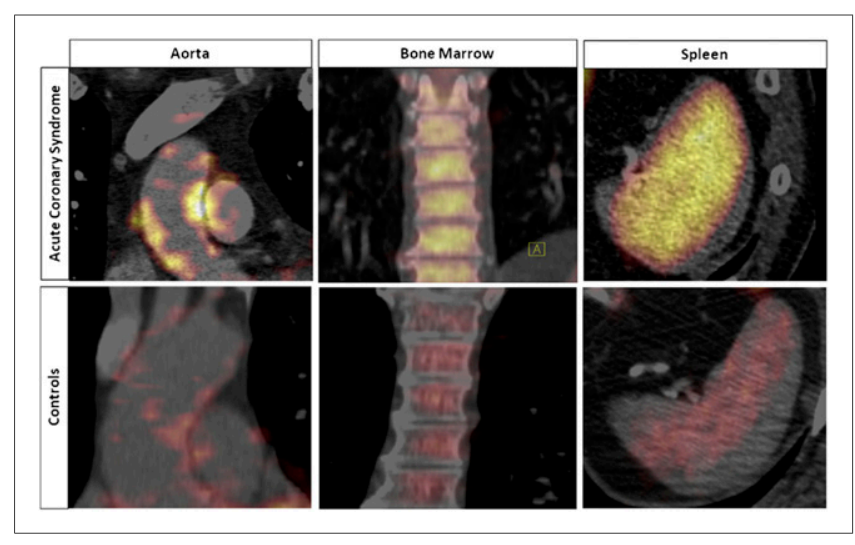

FIGURE 1. Evidence of cardiosplenic axis in ACS patients. ${ }^{18} \mathrm{~F}-\mathrm{FDG}$ uptake is significantly increased in aortic wall (left), bone marrow (middle), and spleen (right) in ACS patients compared with controls. (Reprinted with permission of Emami et al. (9).)

tivity experienced more cardiac events (hazard ratio, 3.3; $P=0.003$ ), even after adjustment for CVD risk factors. These results support the clinical importance of the cardiosplenic axis in humans, and motivate CVD preventative strategies against proinflammatory leukocyte emigration.

New Clinical Disease Insights in Atherosclerosis. Plaque inflammation drives atherosclerosis progression and clinical events, as evidenced by heightened CVD in patients with inflammatory rheumatologic diseases. To examine this association, Naik et al. performed ${ }^{18} \mathrm{~F}-\mathrm{FDG}$ PET in 60 psoriasis patients without known CVD and found that aortic ${ }^{18}$ F-FDG uptake increased proportionately to psoriasis disease severity, even after adjustment for age, sex, and calculated FRS ( $\beta=0.41, P=0.001)(10)$. A separate study of 38 familial hypercholesterolemia subjects who underwent serial ${ }^{18}$ F-FDG PET imaging at baseline (Fig. 2) and then $3 \mathrm{~d}$ later after lipoprotein apheresis was performed (11). Apheresis-based low-density lipoprotein reduction $(284 \pm 118$ vs. $127 \pm 50 \mathrm{mg} / \mathrm{dL}, P<0.001)$ rapidly lowered arterial ${ }^{18} \mathrm{~F}$-FDG activity (target-to-background ratio, $2.05 \pm 0.31$ before vs. $1.91 \pm 0.33$ after; $P<0.02$ ). This intriguing data inform that atherogenic lipoproteininduced arterial inflammation may be quickly reversible, supporting potent lipid therapy use in ACS.

Evaluation of Antiatherosclerosis Pharmacotherapies. Because arterial inflammation remains an important pharmacotherapeutic target, several studies have recently harnessed ${ }^{18}$ F-FDG PET to assess the in vivo antiinflammatory effects of novel atherosclerosis pharmacotherapies. Thus far, there exist 5 compound classes where both clinical endpoint and PET imaging data are available. For 2 of these 5 drug classes (statins and thiazolidinediones), imaging and clinical endpoint trial results have been concordantly positive $(12,13)$. On the other hand, for 3 of 5 drug classes (cholesteryl ester transfer protein inhibitor, lipoproteinassociated phospholipase A2 inhibitor, and P38 MAP kinase inhibitors), imaging and clinical endpoint trials have been 


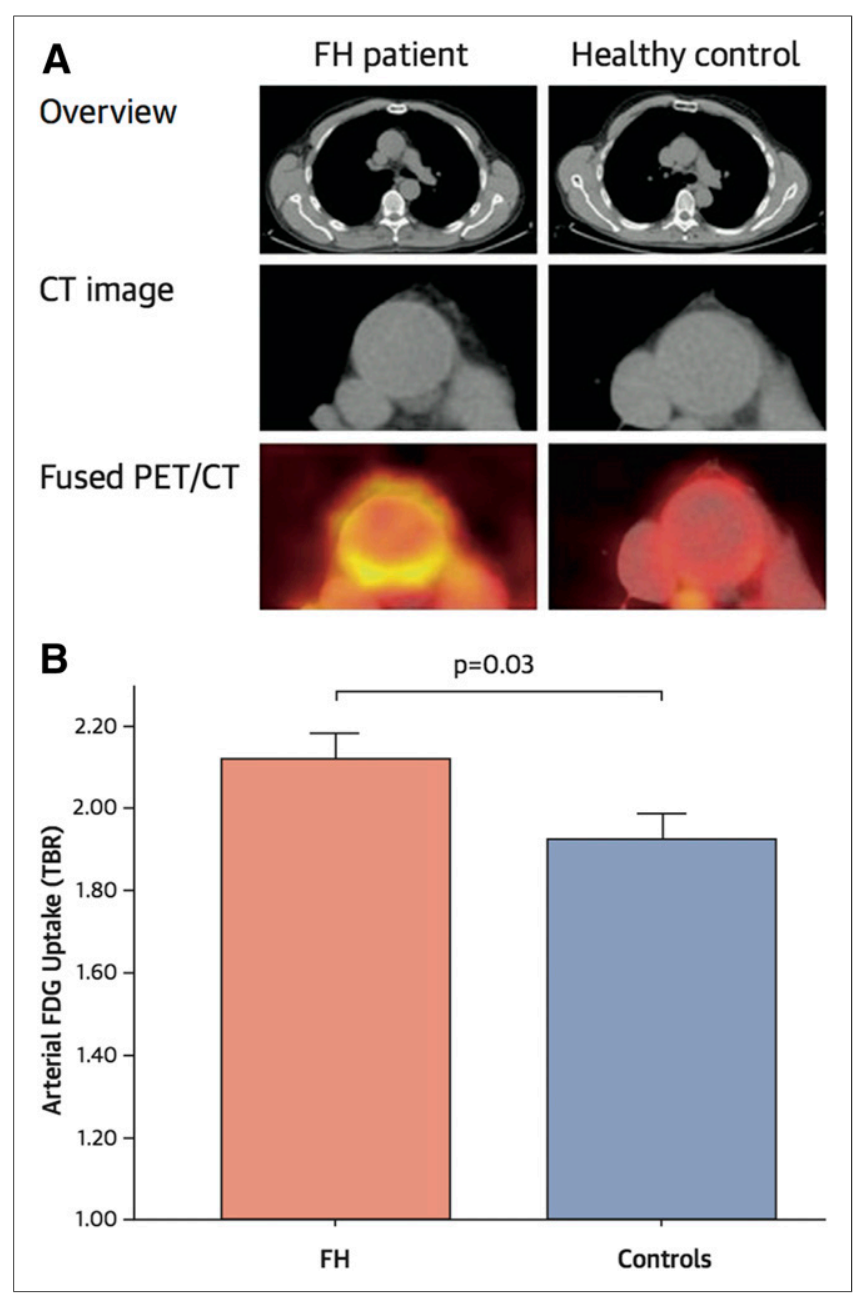

FIGURE 2. ${ }^{18} \mathrm{~F}-\mathrm{FDG} P E T / C T$ inflammation imaging in familial hypercholesterolemia patients. (A) ${ }^{18} \mathrm{~F}-\mathrm{FDG}$ uptake is elevated in the aorta of familial hypercholesterolemia subjects (left) compared with controls (right), as quantified by significantly higher mean arterial target-to-background ratio. $\mathrm{FH}=$ familial hypercholesterolemia; TBR = target-to-background ratio. (Reprinted with permission from van Wijk et al. (11).)

concordantly neutral (14-18). Hence, thus far there appears to be concordance between directional changes in arterial imaging and clinical efficacy. Furthermore, ${ }^{18} \mathrm{~F}-\mathrm{FDG}$ PET/ CT trials are typically small (roughly $35-60$ subjects per group) and fast (3- to 6-mo treatment intervals), and thus have the potential to presage pharmacotherapeutic efficacy in considerably longer and more expensive outcomes trials of several thousand individuals.

\section{Intravascular NIRF Molecular Imaging}

Intravascular NIRF is an emerging optical molecular imaging approach designed for high-resolution coronary arterial imaging. NIRF imaging uses injectable fluorescent imaging agents that target key cellular and molecular processes in vivo. Not only does near-infrared light ( 700-900 nm excitation) offer greater tissue penetration and reduced autofluorescence compared with visible light, but also NIRF imaging can be performed via coro- nary arterial-compatible catheters, and through blood without flushing. These attributes support intravascular NIRF as a viable coronary arterial molecular imaging method.

Jaffer et al. engineered and tested a fully automated rotational 2-dimensional NIRF imaging catheter in rabbit atheroma to detect NIRF inflammatory cysteine protease activity with nanomolar sensitivity (19). Stent-induced inflammatory vascular injury was also demonstrated, suggesting the potential to understand how inflammation may predict restenosis in living subjects. Translationally, indocyanine green (ICG), a Food and Drug Administration-approved NIRF imaging agent, has shown promise for intravascular NIRF imaging (20). A clinical ICG study recently reported that ICG serves as a human in vivo plaque imaging agent in carotid endarterectomy specimens (21). ICG was reliably detected by ex vivo NIRF imaging and near-infrared fluorescence microscopy, and was found to report on endothelial barrier compromise, and deposited in adjacent areas of macrophage infiltration and plaque hemorrhage (Fig. 3).

Because standalone NIRF imaging is limited by a lack of anatomic information, a next-generation intravascular NIRF imaging system incorporating coregistered optical coherence tomography (OCT) structural imaging was constructed (22). Integrated NIRF OCT imaging combines high-sensitivity NIRF detection with exquisite 10 - to $20-\mu \mathrm{m}$ OCT spatial resolution and can illuminate inflammatory plaque protease activity in experimental atherosclerosis, as well as fibrin deposition on clinical-grade coronary stents (23). Recently, first-in-human intracoronary NIRF OCT imaging was successfully performed to image NIR autofluorescence in 12 patients (24). With additional development, including integrating NIRF with intravascular ultrasound (25), intravascular NIRF imaging is positioned to transform clinical molecular imaging of coronary atherosclerosis and stents.

\section{VTE}

\section{Background}

Deep venous thrombosis (DVT) and pulmonary embolism (PE), collectively known as VTE, remain leading causes of morbidity and mortality worldwide. Diagnostically, compression ultrasonography and CT pulmonary angiography are the primary clinical imaging modalities for assessing DVT and PE, respectively. Although ultrasound and CT pulmonary angiography are well validated for diagnosing VTE, they have limited ability to diagnose recurrent VTE, predict the efficacy of fibrinolytic therapy for $\mathrm{PE}$, or predict debilitating complications such as the DVT-induced postthrombotic syndrome, or PE-induced chronic thromboembolic pulmonary hypertension.

VTE is an intertwined thrombotic and inflammatory process, consisting of an initial neutrophil infiltrate followed by a monocyte-macrophage influx. These cells coordinate to promote endogenous fibrinolysis, thrombus stabilization, and 


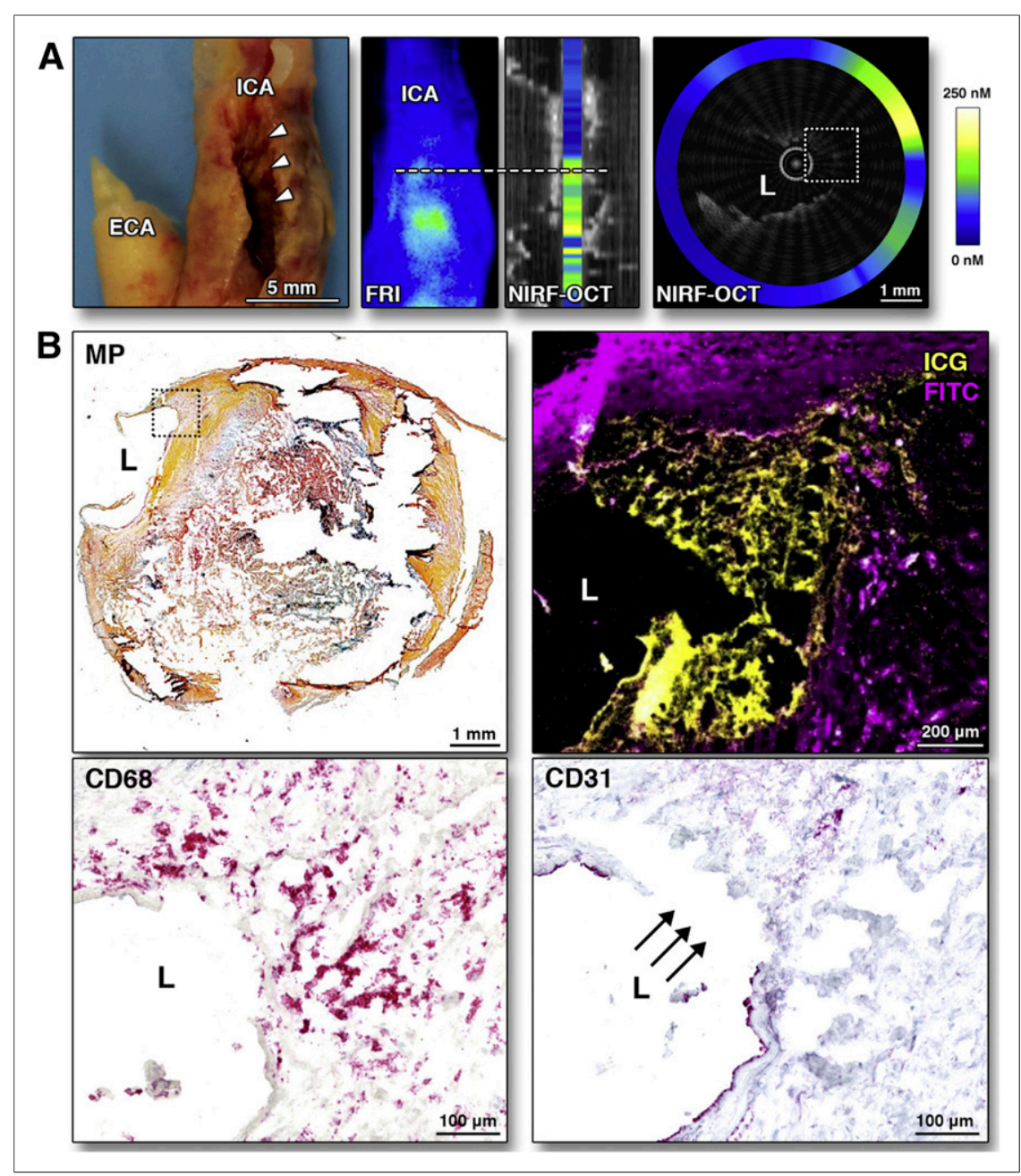

FIGURE 3. NIRF imaging of ICG deposition in human atheroma. (A) Aligned photo, fluorescence reflectance image (FRI), and long-view NIRF OCT fusion image of resected human carotid atheroma. Ninety minutes after intravenous ICG administration, elevated ICG signal (green-yellow) presents area of severe stenosis (arrowheads). NIRF OCT cross-sectional image at white dotted line shows thinned/absent fibrous cap (white dotted box), with corresponding high NIRF ICG signal. (B) Highmagnification histology reveals complex plaque (top left, Movat's Pentachrome [MP]) and surface ICG NIRF uptake (yellow, top right; fluorescein isothiocyanate [FITC autofluorescence (purple)]). ICG NIRF-positive areas demonstrate endothelial barrier disruption (CD31; arrows) and macrophage infiltration (CD68). ICA = internal carotid artery; $L=$ lumen. (Reprinted with permission from Verjans et al. (21).)

first-time (primary) DVT. The authors observed significantly higher ${ }^{18} \mathrm{~F}-\mathrm{FDG}$ uptake in thrombosed vein segments over matched contralateral segments without thrombosis. ${ }^{18} \mathrm{~F}-\mathrm{FDG}$ was accurate in diagnosing DVT with $87.5 \%$ sensitivity and $100 \%$ specificity. Although limited by a small sample size, this study demonstrated that the ${ }^{18} \mathrm{~F}$ FDG DVT uptake diminished over time (26).

Recurrent DVT. Recurrent DVT occurs up to $10 \%$ per year after an unprovoked DVT and increases the risk of postthrombotic syndrome, PE, and death (27). Although accurate diagnosis of recurrent same-site VTE is critical for justifying reinitiation of anticoagulation therapy, its diagnosis is challenging for conventional ultrasound due to residual vein wall scarring or thrombus from the initial DVT. Recognizing that ${ }^{18}$ F-FDG PET can report on DVT metabolic or inflammatory activity, and that a recurrent DVT should generate a new inflammatory response, Hara et al. leveraged ${ }^{18} \mathrm{~F}-\mathrm{FDG}$ PET/CT to accurately diagnose recurrent DVT in mice (Fig. 4) (28). Analysis of thrombosed veins showed that most ${ }^{18} \mathrm{~F}-\mathrm{FDG}$ uptake was in the thrombus $(70 \%)$, with a minority signal in the vein wall $(30 \%)$. Furthermore, they observed that inflammatory ${ }^{18}$ F-FDG DVT signal was more closely associated with neutrophils than macrophages and that ${ }^{18} \mathrm{~F}$-FDG uptake was neutrophil-dependent and accordingly higher in early-stage, neutrophil-rich DVT. Finally, the authors demonstrated that clinical ${ }^{18} \mathrm{~F}-\mathrm{FDG}$ uptake in DVT diminishes with increasing DVT age, extending earlier results.

ensuing VTE resolution. Several thrombus-targeted imaging agents have been developed for PET or SPECT imaging (Table 1) but have not yet demonstrated diagnostic advantages over anatomic ultrasound or CT imaging. However, VTE inflammation as an imaging target has undergone limited evaluation. Recently, ${ }^{18}$ F-FDG PET/CT imaging of VTE has been used in humans to assess DVT age. Future studies are needed to assess this tool's role in diagnosing recurrent DVT, a vexing clinical problem.

\section{${ }^{18} \mathrm{~F}-\mathrm{FDG}$ PET Imaging of VTE}

Primary DVT. Rondina et al. performed the first prospective study demonstrating that ${ }^{18} \mathrm{~F}-\mathrm{FDG} \mathrm{PET} / \mathrm{CT}$ is a sensitive imaging tool for detecting symptomatic, proximal
$P E$. Case reports and retrospective studies have described ${ }^{18} \mathrm{~F}-\mathrm{FDG}$ uptake in PE, mostly as incidental findings in whole-body cancer staging ${ }^{18}$ F-FDG PET/CT imaging sessions (29-31). As suspected, metabolically active tumors may mimic VTE ${ }^{18}$ F-FDG enhancement, increasing the chances of false-positive diagnosis $(32,33)$. Interestingly, delayed ${ }^{18} \mathrm{~F}$-FDG imaging ( $2 \mathrm{~h}$ after injection) may be more specific for PE and should be considered in future studies (30). Although CT pulmonary angiography is the gold standard for PE diagnosis, ${ }^{18}$ F-FDG PET might also be useful for detecting recurrent PE (28). In addition, ${ }^{18} \mathrm{~F}-\mathrm{FDG}$ PET assessment of thrombus age could help predict the success of fibrinolytic therapies, as suggested by recent preclinical molecular imaging investigations $(34,35)$. 
TABLE 1

Selected Clinical and Translational Metabolic and Molecular Imaging Agents for Illuminating Atherosclerosis and VTE

\begin{tabular}{|c|c|c|c|c|c|}
\hline Agent & Application & Target & Modality & $\begin{array}{l}\text { Clinically } \\
\text { tested? }\end{array}$ & Reference \\
\hline \multirow[t]{2}{*}{${ }^{18} \mathrm{~F}-\mathrm{FDG}$} & Atherosclerosis & $\begin{array}{l}\text { Macrophages, } \\
\text { hypoxia }\end{array}$ & PET & Yes & Circulation. 2002;105:2708-2711 \\
\hline & & & & & J Am Coll Cardiol. 2011;58:603-614 \\
\hline \multirow[t]{2}{*}{${ }^{18} \mathrm{~F}$-sodium fluoride } & Atherosclerosis & Calcification & PET & Yes & J Nucl Med. 2015;56:1019-1023 \\
\hline & & & & & Lancet. 2014;383:705-713 \\
\hline${ }^{18} \mathrm{~F}$-fluorothymidine & Atherosclerosis & Cell proliferation & PET & Yes & Circ Res. 2015;117:835-845 \\
\hline${ }^{18} \mathrm{~F}$-fluorocholine & Atherosclerosis & Cell proliferation & PET & Yes & Circ Cardiovasc Imaging. 2016;9:e004467 \\
\hline \multirow[t]{2}{*}{${ }^{68} \mathrm{Ga}$-dotatate } & Atherosclerosis & $\begin{array}{l}\text { Somatostatin } \\
\text { receptors }\end{array}$ & PET & Yes & J Am Coll Cardiol. 2013;62:2344-2345 \\
\hline & & & & & J Am Coll Cardiol. 2017;69:1774-1791 \\
\hline${ }^{68} \mathrm{Ga}$-pentixafor & Atherosclerosis & $\begin{array}{l}\text { Macrophage } \\
\text { CXC4 receptors }\end{array}$ & PET & Yes & J Nucl Med. Oct 27, 2016 [Epub ahead of print] \\
\hline${ }^{18} \mathrm{~F}$-galactoRGD & Atherosclerosis & $\alpha_{v} \beta_{3}$ integrins & PET & Yes & JACC Cardiovasc Imaging. 2014;7:178-187 \\
\hline${ }^{64} \mathrm{Cu}-\mathrm{ATSM}$ & Atherosclerosis & Macrophages & PET & Yes & J Nucl Med. 2016;57:2006-2011 \\
\hline${ }^{64} \mathrm{Cu}$-CANFComb & Atherosclerosis & Macrophages & PET & Yes & J Nucl Med. 2010;51:85-91 \\
\hline USPIO & Atherosclerosis & Macrophages & MRI & Yes & Circulation. 2003;107:2453-2458 \\
\hline ICG & Atherosclerosis & $\begin{array}{l}\text { Endothelial } \\
\text { permeability }\end{array}$ & NIRF & Yes & JACC Cardiovasc Imaging. 2016;9:1087-1095 \\
\hline \multirow[t]{2}{*}{ 99mTc-apcitide } & VTE & $\begin{array}{l}\text { Platelet glycoprotein } \\
\text { Ilb/IIla receptors }\end{array}$ & Scintigraphy & Yes & J Nucl Med. 1995;36:1384-1391 \\
\hline & & & & & J Nucl Med. 2000;41:1214-1223 \\
\hline \multirow[t]{2}{*}{ 99mTc-DMP444 } & VTE & $\begin{array}{l}\text { Platelet glycoprotein } \\
\text { IIb/IIla receptors }\end{array}$ & Scintigraphy & Yes & Coron Artery Dis. 1998;9:131-141 \\
\hline & & & & & J Nucl Cardiol. 2000;7:359-364 \\
\hline \multirow[t]{2}{*}{${ }^{111} \ln -59 D 8$} & VTE & Fibrin & Scintigraphy & Yes & Science. 1983;222:1129-1132 \\
\hline & & & & & J Nucl Med. 1991;32:785-791 \\
\hline \multirow[t]{5}{*}{ EP-2104R/FTP11 } & $\begin{array}{l}\text { Atherosclerosis, } \\
\text { VTE }\end{array}$ & Fibrin & $\begin{array}{l}\text { MRI/SPECT } \\
\text { NIRF }\end{array}$ & Yes/no & Eur Radiol. 2008;18:1995-2005 \\
\hline & & & & & Contrast Medial Mol Imaging. 2013;8:229-237 \\
\hline & & & & & JACC Cardiovasc Imaging. 2012;5:607-615 \\
\hline & & & & & Circulation. 2014;130:1044-1052 \\
\hline & & & & & Theranostics. 2015;5:1317-1327 \\
\hline Prosense VM110 & Atherosclerosis & $\begin{array}{l}\text { Cathepsin } \\
\text { proteases }\end{array}$ & NIRF & No & J Am Coll Cardiol. 2011;57:2516-2526 \\
\hline MMR-Cy7 & Atherosclerosis & $\begin{array}{l}\text { Macrophage } \\
\text { mannose receptors }\end{array}$ & NIRF & No & Sci Rep. 2016;6:22608 \\
\hline${ }^{18} \mathrm{~F}$-fluorodeoxymannose & Atherosclerosis & $\begin{array}{l}\text { Macrophage } \\
\text { mannose receptors }\end{array}$ & PET & No & Nat Med. 2014;20:215-219 \\
\hline
\end{tabular}

\section{SUMMARY AND FUTURE DIRECTIONS}

\section{Atherosclerosis}

As showcased above, metabolic and molecular imaging approaches have the potential to better predict clinical events, tailor selection of pharmacotherapeutic and interventional therapies, and assess pharmaceutical efficacy.

Large Arterial Beds. Larger arteries, in particular the carotid artery, are well suited to noninvasive PET imaging. Therefore, we anticipate that future studies of carotid ${ }^{18} \mathrm{~F}$-FDG PET will extend the initial insights provided by Marnane et al. (6), and better determine which carotid lesions are high risk and warrant a more intensive medical or interventional approach. This is an area of vital importance, as there remains controversy about when to intervene on asymptomatic severe interval carotid artery lesions, and whether biologic data (beyond currently available stenosis information) might help guide that decision (36). Risk-benefit of radiation exposure and costeffectiveness will need to be further analyzed.

In addition to assessing plaque inflammation in clinical subjects, as robustly demonstrated $(4,5,7-9,11,12,14,18)$, noninvasive carotid ${ }^{18} \mathrm{~F}$-FDG PET/CT will continue to play a role in evaluating novel pharmacotherapy antiinflammatory effects. New tracers have an opportunity to improve the ability to sensitively and specifically detect plaque inflammation (37) but will need outcome validation to serve as a compelling challenger to ${ }^{18} \mathrm{~F}-\mathrm{FDG}$.

Coronary Arterial Imaging. Background cardiomyocyte metabolic signal, cardiorespiratory motion, and partial-volume effects greatly affect the reliability to detect ${ }^{18}$ F-FDG signal in 


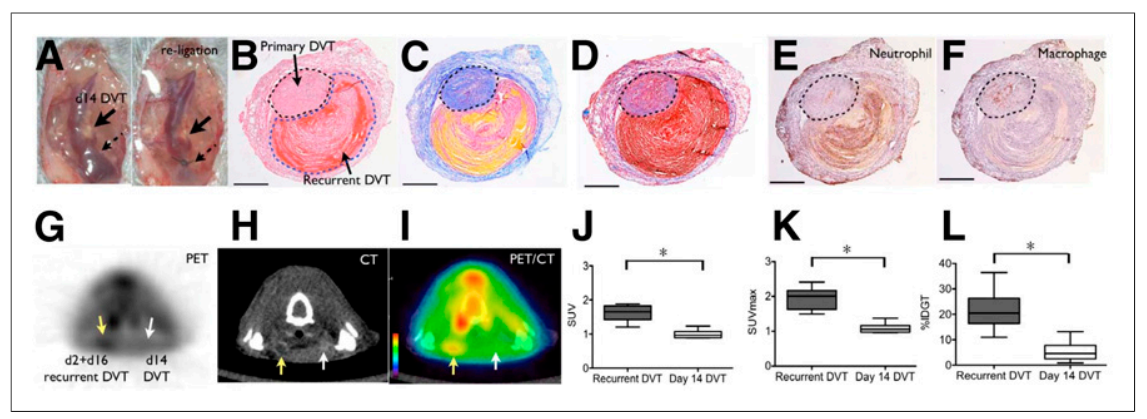

FIGURE 4. ${ }^{18} \mathrm{~F}-\mathrm{FDG}$ PET/CT imaging of recurrent VTE. (A) Mouse model illustrating day-14 DVT and religation of jugular vein to create recurrent DVT mouse model used in ${ }^{18}$ F-FDG PET imaging. Histologic staining with hematoxylin and eosin (B), Carstairs (C), and Masson's Trichrome (D) of resected day-2 recurrent DVT (black dotted line) overlying day-16 DVT (blue dotted line) show red blood cell and fibrin-rich areas in recurrent DVT (red and yellow area in B-D) and collagen-rich zones in older primary DVT (blue areas in $C$ and D). Immunostaining for neutrophils $(E)$ and macrophages $(F)$ highlight differences between recurrent DVT and older DVT. ${ }^{18} \mathrm{~F}-\mathrm{FDG}$ PET/CT images (G-I) of recurrent and primary DVT show increased ${ }^{18} \mathrm{~F}-\mathrm{FDG}$ uptake in recurrent DVT (J-L). Yellow arrows = recurrent DVT; white arrows = primary day 16 DVT. (Reprinted with permission from Hara et al. (28).)

coronary arteries, particularly distal to the proximal coronary segments. In contrast, ${ }^{18} \mathrm{~F}-\mathrm{Na}$ PET does not suffer from myocardial background uptake and appears to be a reliable noninvasive option for imaging coronary plaque osteogenic activity/calcification. Future studies are needed to determine ${ }^{18} \mathrm{~F}-\mathrm{Na}$ 's clinical value beyond widely available calcium scoring and CT angiography. There is also promise for this tracer to understand the potential effects of bisphosphonates and statins on the pathophysiology of coronary calcification.

Intravascular NIRF molecular imaging shows promise for high-resolution molecular imaging and can be integrated with intravascular ultrasound (25) or OCT (22), strengthening the ability to provide comprehensive molecular-structural imaging of atherosclerosis and stent biology. However, NIRF imaging is in its infancy for clinical translation and will require clinical outcome studies to determine its value. Given its invasive requirement, NIRF imaging will likely be used to further stratify patients already undergoing percutaneous coronary intervention for ACS or stable angina.

\section{VTE}

The ability of ${ }^{18}$ F-FDG PET to image VTE inflammation may provide a new opportunity to detect recurrent DVT and may provide a foundation for guiding anticoagulation decisions. However, outcome and safety studies are needed. In addition, ${ }^{18} \mathrm{~F}$-FDG PET may soon provide insights into the postthrombotic syndrome, a common complication of DVT that is partly inflammation driven. For PE, new imaging tracers may help identify which thrombi are amenable to fibrinolysis, a question of particular importance given recent neutral data of fibrinolysis in patients with intermediate-risk PE (38).

\section{CONCLUSION}

Metabolic and molecular imaging with ${ }^{18} \mathrm{~F}-\mathrm{FDG}$ PET and other modalities are providing valuable insights into athero- sclerosis and VTE pathophysiology in human subjects. ${ }^{18} \mathrm{~F}-\mathrm{FDG}$ PET and emerging metabolic/molecular imaging technologies are expected to evolve as outcomes studies emerge, and are further analyzed for cost-effectiveness and safety. Overall, these advances are expected to shed important insight into atherosclerotic disease pathophysiology, help identify novel treatment strategies, and improve clinical evaluation and management of individual patients.

\section{DISCLOSURE}

The following sources supported this study: NIH R01HL122388 (Farouc A. Jaffer), R01HL122177 (Ahmed Tawakol), and K08HL130465 (Eric A. Osborn); and MGH Hassenfeld Research Scholar Fund (Farouc A. Jaffer). Dr. Jaffer received research funding from Siemens and Canon and has a consulting agreement with Boston Scientific and Abbott Vascular. Massachusetts General Hospital has a patent licensing arrangement with Canon Corporation. Dr. Jaffer has the right to receive licensing royalties through this licensing arrangement. Dr. Osborn has consulting agreements with DynaMed and St. Jude Medical. Dr. Tawakol received research funding from Actelion, Genetech, and Takeda and has a consulting agreement with Actelion. No other potential conflict of interest relevant to this article was reported.

\section{REFERENCES}

1. Noh TS, Moon SH, Cho YS, et al. Relation of carotid artery ${ }^{18} \mathrm{~F}-\mathrm{FDG}$ uptake to C-reactive protein and Framingham risk score in a large cohort of asymptomatic adults. J Nucl Med. 2013;54:2070-2076.

2. van der Valk FM, Bekkering S, Kroon J, et al. Oxidized phospholipids on lipoprotein(a) elicit arterial wall inflammation and an inflammatory monocyte response in humans. Circulation. 2016;134:611-624

3. Cho SG, Park KS, Kim J, et al. Prediction of coronary artery calcium progression by FDG uptake of large arteries in asymptomatic individuals. Eur J Nucl Med Mol Imaging. 2017;44:129-140.

4. Abdelbaky A, Corsini E, Figueroa AL, et al. Focal arterial inflammation precedes subsequent calcification in the same location: a longitudinal FDGPET/CT study. Circ Cardiovasc Imaging. 2013;6:747-754.

5. Figueroa AL, Abdelbaky A, Truong QA, et al. Measurement of arterial activity on routine FDG PET/CT images improves prediction of risk of future CV events. JACC Cardiovasc Imaging. 2013;6:1250-1259.

6. Marnane M, Merwick A, Sheehan OC, et al. Carotid plaque inflammation on ${ }^{18} \mathrm{~F}$-fluorodeoxyglucose positron emission tomography predicts early stroke recurrence. Ann Neurol. 2012;71:709-718.

7. Figueroa AL, Takx RA, MacNabb MH, et al. Relationship between measures of adiposity, arterial inflammation, and subsequent cardiovascular events. Circ Cardiovasc Imaging. 2016;9:e004043.

8. Takx RA, Ishai A, Truong QA, MacNabb MH, Scherrer-Crosbie M, Tawakol A. Supraclavicular brown adipose tissue ${ }^{18}$ F-FDG uptake and cardiovascular disease. J Nucl Med. 2016;57:1221-1225.

9. Emami H, Singh P, MacNabb M, et al. Splenic metabolic activity predicts risk of future cardiovascular events: demonstration of a cardiosplenic axis in humans. JACC Cardiovasc Imaging. 2015;8:121-130. 
10. Naik HB, Natarajan B, Stansky E, et al. Severity of psoriasis associates with aortic vascular inflammation detected by FDG PET/CT and neutrophil activation in a prospective observational study. Arterioscler Thromb Vasc Biol. 2015;35:2667-2676.

11. van Wijk DF, Sjouke B, Figueroa A, et al. Nonpharmacological lipoprotein apheresis reduces arterial inflammation in familial hypercholesterolemia. J Am Coll Cardiol. 2014;64:1418-1426.

12. Tawakol A, Fayad ZA, Mogg R, et al. Intensification of statin therapy results in a rapid reduction in atherosclerotic inflammation: results of a multicenter fluorodeoxyglucosepositron emission tomography/computed tomography feasibility study. J Am Coll Cardiol. 2013;62:909-917.

13. Mizoguchi M, Tahara N, Tahara A, et al. Pioglitazone attenuates atherosclerotic plaque inflammation in patients with impaired glucose tolerance or diabetes a prospective, randomized, comparator-controlled study using serial FDG PET/CT imaging study of carotid artery and ascending aorta. JACC Cardiovasc Imaging. 2011;4:1110-1118.

14. Emami H, Vucic E, Subramanian S, et al. The effect of BMS-582949, a P38 mitogen-activated protein kinase (P38 MAPK) inhibitor on arterial inflammation: a multicenter FDG-PET trial. Atherosclerosis. 2015;240:490-496.

15. STABILITY Investigators, White HD, Held C, et al. Darapladib for preventing ischemic events in stable coronary heart disease. N Engl J Med. 2014;370:17021711.

16. O'Donoghue ML, Glaser R, Cavender MA, et al. Effect of losmapimod on cardiovascular outcomes in patients hospitalized with acute myocardial infarction: a randomized clinical trial. JAMA. 2016;315:1591-1599.

17. Schwartz GG, Olsson AG, Abt M, et al. Effects of dalcetrapib in patients with a recent acute coronary syndrome. N Engl J Med. 2012;367:2089-2099.

18. Tawakol A, Singh P, Rudd JH, et al. Effect of treatment for 12 weeks with rilapladib, a lipoprotein-associated phospholipase A2 inhibitor, on arterial inflammation as assessed with ${ }^{18} \mathrm{~F}$-fluorodeoxyglucose-positron emission tomography imaging. $J$ Am Coll Cardiol. 2014;63:86-88.

19. Jaffer FA, Calfon MA, Rosenthal A, et al. Two-dimensional intravascular nearinfrared fluorescence molecular imaging of inflammation in atherosclerosis and stent-induced vascular injury. J Am Coll Cardiol. 2011;57:2516-2526.

20. Vinegoni C, Botnaru I, Aikawa E, et al. Indocyanine green enables near-infrared fluorescence imaging of lipid-rich, inflamed atherosclerotic plaques. Sci Transl Med. 2011;3:84ra45.

21. Verjans JW, Osborn EA, Ughi GJ, et al. Targeted near-infrared fluorescence imaging of atherosclerosis: clinical and intracoronary evaluation of indocyanine green. JACC Cardiovasc Imaging. 2016;9:1087-1095.

22. Yoo H, Kim JW, Shishkov M, et al. Intra-arterial catheter for simultaneous microstructural and molecular imaging in vivo. Nat Med. 2011;17:1680-1684.

23. Hara T, Ughi GJ, McCarthy JR, et al. Intravascular fibrin molecular imaging improves the detection of unhealed stents assessed by optical coherence tomography in vivo. Eur Heart J. December 18, 2015 [Epub ahead of print].
24. Ughi GJ, Wang H, Gerbaud E, et al. Clinical characterization of coronary atherosclerosis with dual-modality OCT and near-infrared autofluorescence imaging. JACC Cardiovasc Imaging. 2016;9:1304-1314.

25. Bozhko D, Osborn EA, Rosenthal A, et al. Quantitative intravascular biological fluorescence-ultrasound imaging of coronary and peripheral arteries in vivo. Eur Heart J Cardiovasc Imaging. December 27, 2016 [Epub ahead of print].

26. Rondina MT, Lam UT, Pendleton RC, et al. ${ }^{18} \mathrm{~F}-\mathrm{FDG}$ PET in the evaluation of acuity of deep vein thrombosis. Clin Nucl Med. 2012;37:1139-1145.

27. Lecumberri R, Alfonso A, Jimenez D, et al. Dynamics of case-fatality rates of recurrent thromboembolism and major bleeding in patients treated for venous thromboembolism. Thromb Haemost. 2013;110:834-843.

28. Hara T, Truelove J, Tawakol A, et al. ${ }^{18} \mathrm{~F}$-fluorodeoxyglucose positron emission tomography/computed tomography enables the detection of recurrent same-site deep vein thrombosis by illuminating recently formed, neutrophil-rich thrombus. Circulation. 2014;130:1044-1052.

29. Khandelwal AR, Li G, Takalkar AM. Incidental detection of unsuspected pulmonary embolism on oncologic FDG PET/CT imaging. Clin Nucl Med. 2011;36:720-722.

30. Gorur GD, Ciftci E, Isgoren S, Demir H, Haksal C. Pulmonary ${ }^{18}$ F-FDG microembolism detected on only delayed image in a PET/CT study. Clin Nucl Med. 2015;40:253-255.

31. Hess S, Madsen PH, Iversen ED, Frifelt JJ, Hoilund-Carlsen PF, Alavi A. Efficacy of FDG PET/CT imaging for venous thromboembolic disorders: preliminary results from a prospective, observational pilot study. Clin Nucl Med. 2015;40:e23-e26.

32. Kessler A, Son H. Pulmonary artery angiosarcoma on ${ }^{18}$ F-FDG PET/CT masquerading as pulmonary embolism. Clin Nucl Med. 2015;40:82-84.

33. Guo Y, Wang T, Yang M. Pulmonary artery sarcoma detected on ${ }^{18} \mathrm{~F}-\mathrm{FDG}$ PET/ CT with unusual findings. Clin Nucl Med. 2015;40:e530-e531.

34. Andia ME, Saha P, Jenkins J, et al. Fibrin-targeted magnetic resonance imaging allows in vivo quantification of thrombus fibrin content and identifies thrombi amenable for thrombolysis. Arterioscler Thromb Vasc Biol. 2014;34:1193-1198.

35. Stein-Merlob AF, Kessinger CW, Erdem SS, et al. Blood accessibility to fibrin in venous thrombosis is thrombus age-dependent and predicts fibrinolytic efficacy: an in vivo fibrin molecular imaging study. Theranostics. 2015;5:1317-1327.

36. Chaturvedi S, Chimowitz M, Brown RD Jr, Lal BK, Meschia JF. The urgent need for contemporary clinical trials in patients with asymptomatic carotid stenosis. Neurology. 2016;87:2271-2278.

37. Tarkin JM, Joshi FR, Evans NR, et al. Detection of atherosclerotic inflammation by ${ }^{68} \mathrm{Ga}$-DOTATATE PET compared to $\left[{ }^{18} \mathrm{~F}\right] \mathrm{FDG}$ PET imaging. $J$ Am Coll Cardiol. 2017;69:1774-1791.

38. Konstantinides SV, Vicaut E, Danays T, et al. Impact of thrombolytic therapy on the long-term outcome of intermediate-risk pulmonary embolism. J Am Coll Cardiol. 2017;69:1536-1544 